\title{
Photochemistry of 5-Hydroxy-4'-Dimethylaminoflavylium in the Presence of SDS Micelles. The Role of Metastable States of Flavylium Cation-Quinoidal Base and Trans-Chalcones
}

\author{
Paula Araújo, ${ }^{\mathrm{a}}$ Johan Mendoza, ${ }^{\mathrm{b}}$ Fernando Pina, ${ }^{\mathrm{b} *}$ Ana Rita Pereira, ${ }^{\mathrm{a}}$ Iva Fernandes, ${ }^{\mathrm{a}}$ \\ Victor de Freitas, ${ }^{\mathrm{a}}$ Joana Oliveira, ${ }^{\text {a* }}$ \\ ${ }^{a}$ LAQV - REQUIMTE, Departamento de Química e Bioquímica, Faculdade de Ciências, Universidade \\ do Porto, Rua do Campo Alegre, 687, 4169-007 Porto, Portugal, *jsoliveira@ @c.up.pt. \\ ${ }^{\mathrm{b}}$ LAQV - REQUIMTE, Departamento de Química, Faculdade de Ciências e Tecnologia, Universidade \\ Nova de Lisboa, 2829-516 Caparica, Portugal,*fp@ fc.up.pt.
}

\begin{abstract}
The multistate of species originated by the compound 5-hydroxy-4'dimethylaminoflavylium was studied in the presence of sodium dodecyl sulfate micelles $0.1 \mathrm{M}$. A series of $\mathrm{pH}$ jumps obtained by addition of base to the equilibrated solutions of the flavylium cation results in a metastable equilibrium between the flavylium cation and the quinoidal base with $\mathrm{p} K_{\mathrm{a}}=7.6$. In neutral to moderately basic medium, only at $60^{\circ} \mathrm{C}$ and after 5 days a new distribution involving the flavylium cation and trans-chalcones is achieved. On the other hand, the anionic trans-chalcone can be formed in few minutes in very basic medium at room temperature and from this, another meta-stable state constituted by protonated, neutral and anionic transchalcones, $\mathrm{p} K_{\mathrm{Ct}+/ \mathrm{Ct}}=2.6$ and $\mathrm{p} K_{\mathrm{C} t / \mathrm{Ct}-}=10.7$ is reached. The equilibrium is apparently
\end{abstract}


attained between flavylium cation, neutral and anionic trans-chalcones respectively for $\mathrm{p} K_{\mathrm{AH}+/ \mathrm{Ct}}=4.1$ and $\mathrm{p} K_{\mathrm{Ct} / \mathrm{Ct}-}=10.7$.

Irradiation of the neutral (stable or metastable) and protonated (meta-stable) transchalcones yields to the appearance of the quinoidal base or the flavylium cation in an extended $\mathrm{pH}$ region $1<\mathrm{pH}<10$. The peculiar properties of this flavylium based photochromic system are explained by means of an energy level diagram where cischalcone and hemiketal lay above the quinoidal base.

Keywords: Photochromism, Aminoflavylium, Quinoidal base, trans-Chalcones, Meta-stability, Anthocyanins 
Graphical abstract

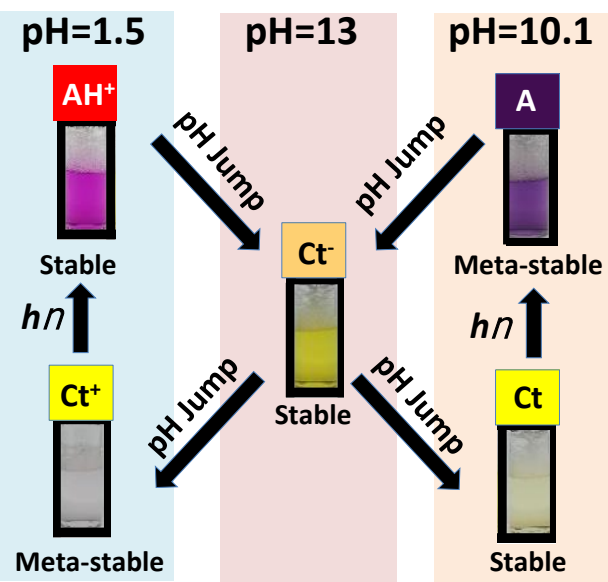




\section{Introduction}

Based on the study of many anthocyanins and related compounds it is possible to predict for any flavylium structure the chemical species that will be generate by increasing the $\mathrm{pH} \cdot{ }^{[1],[2],[3],[4],[5]}$ In the case of the newly synthesized 5-hydroxy-4'dimethylaminoflavylium (5OH4'NMe2) they are shown in Scheme 1. In the experimental conditions used within this work (SDS micelles) no evidence for the dianionic species was achieved and the protonated flavylium cation was only observed at extremely acidic conditions.

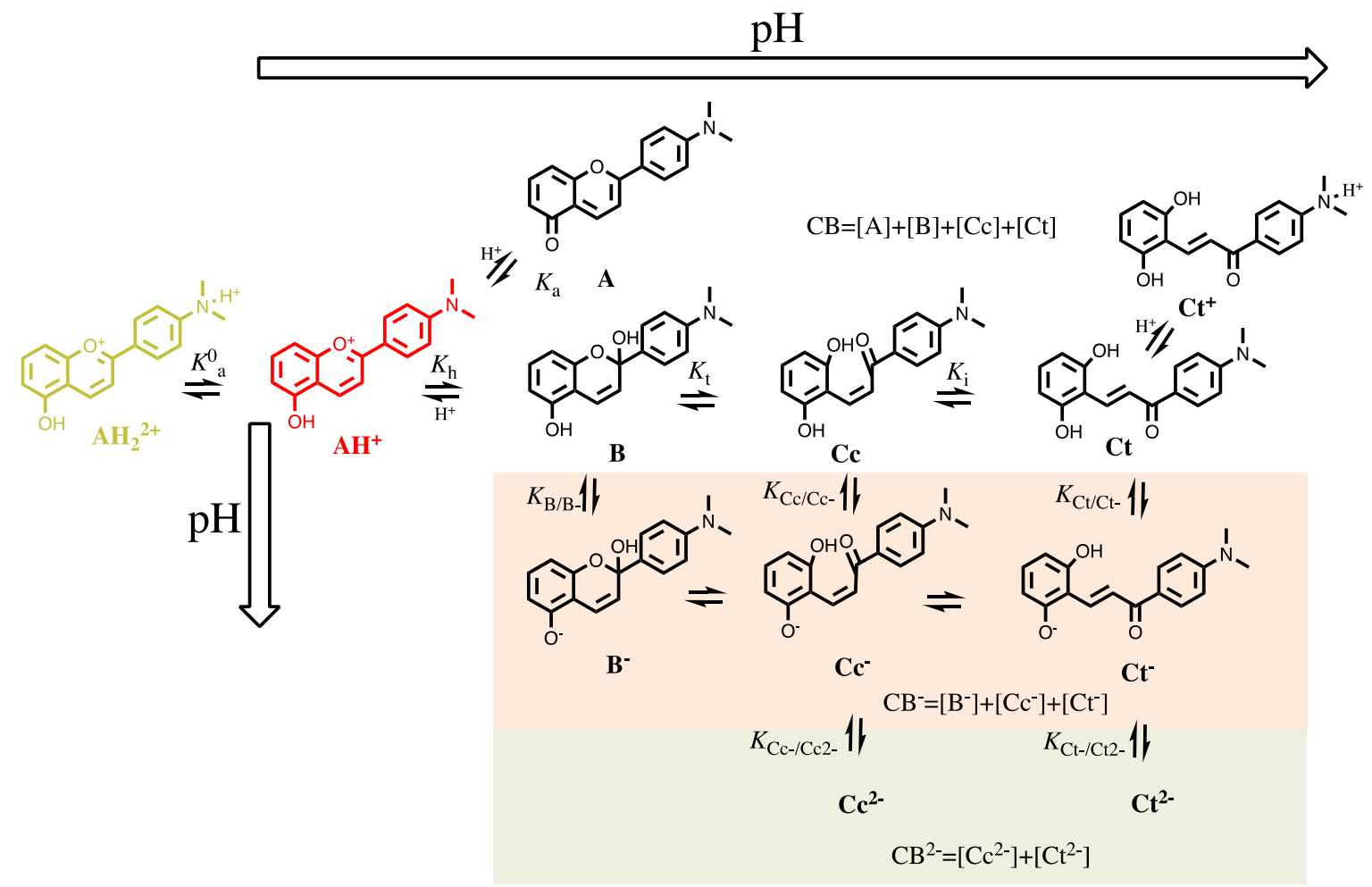


Scheme 1. Multistate of all possible species of 5-hydroxy-4'-dimethylaminoflavylium (5OH4'NMe2).

The flavylium cation $\left(\mathbf{A H}^{+}\right)$gives the name to the multistate and is the only species present at sufficiently acidic medium. ${ }^{a}$ The system is conveniently studied by addition of base to equilibrated solutions of the flavylium cation (direct $\mathrm{pH}$ jumps)[6]:[5] or by addition of acid to equilibrated solutions at higher $\mathrm{pH}$ values, $[4,5]$ in the present work the anionic trans-chalcone (reverse $\mathrm{pH}$ jumps). Upon a direct $\mathrm{pH}$ jump at sufficiently high $\mathrm{pH}$ values the quinoidal base (A) is formed in sub-microseconds.[7] In anthocyanins and related compounds, the evolution of the system towards the equilibrium takes place from the hydration of the flavylium cation to give hemiketal (B) and not from the quinoidal base, which only in basic medium is attacked by $\mathrm{OH}^{-}$ Error! Bookmark not defined..[2] This is a breakthrough discovery made by Brouillard and Dubois and it is essential to the comprehension of the system, in particular to explain why in acidic to moderately acidic medium the hydration rate decreases by $\mathrm{pH}$ increasing. Error! Bookmark not defined. The hemiketal (B) is generally in fast equilibrium (seconds to sub-seconds) with cis-chalcone $(\mathbf{C c})$ through a ring opening/closure process (tautomerization) and trans-chalcone $(\mathbf{C t})$ is formed by an isomerization process from cis-chalcone.

\footnotetext{
${ }^{a}$ Depending on the system, the $\mathrm{pH}$ could be more or less acid.
} 


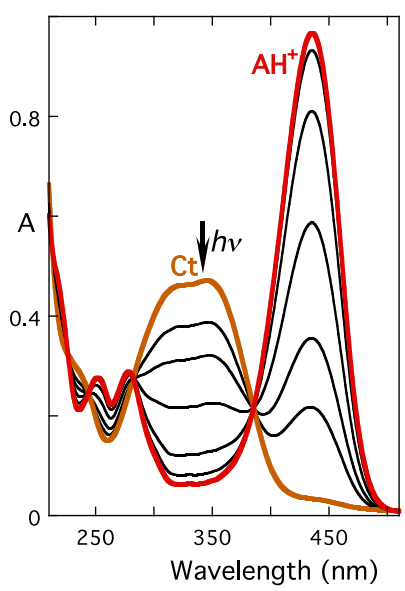

(a)

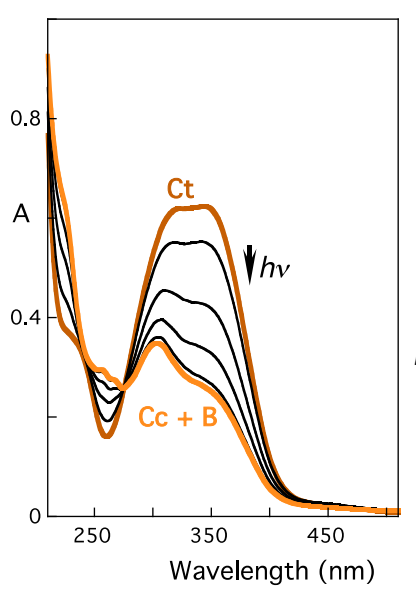

(b)

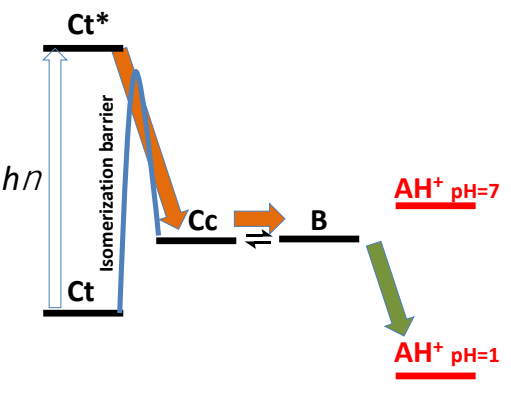

(c)

Figure 1. (a) Spectral variations of 4'-methoxyflavylium at $\mathrm{pH}=1.0$, upon irradiation at $365 \mathrm{~nm}$. (b)

The same at $\mathrm{pH}=7.0$ from the metastable trans-chalcone; (c) Qualitative energy level diagram to account for the photochromic system behaviour.

The photochemistry associated to the multistate of species originated by flavylium compounds has been subjected of interest since many years ago.[8],[9],[10],[11] These systems have been used as models for optical memories capable of write-readerase[12] and even mimic elementary properties of neurons.[13] The photochromic system is illustrated in Fig. 1 for the compound 4'-methoxyflavylium. As shown in Fig. 1c the trans-chalcone is the stable species at higher $\mathrm{pH}$ values, but it is metastable at lower $\mathrm{pHs}$ as for example $\mathrm{pH}=1.0 .{ }^{\mathrm{b}}$ When trans-chalcone is irradiated at $\mathrm{pH}=1$, cis-chalcone is formed and equilibrates in seconds or sub-seconds ${ }^{\mathrm{c}}$ with hemiketal. From this last species the system goes spontaneously to flavylium cation and no reversibility is observed, Fig. 1a (positive photochromism). Conversely, if the stable trans-chalcone is irradiated at $\mathrm{pH}=7$, the flavylium cation is not

\footnotetext{
${ }^{\mathrm{b}}$ The anionic trans-chalcones are the stable species at very basic solutions (for example $\mathrm{pH}=13$ ). The neutral and meta-stable trans-chalcone are obtained by protonation of the anionic species.

${ }^{\mathrm{c}}$ The tautomerization is catalyzed by $\mathrm{H}^{+}$and $\mathrm{OH}^{-}$
} 
thermodynamically accessible and the photoproducts are cis-chalcone in equilibrium with hemiketal, Fig. 1b (negative photochromism). In this case slow reversibility back to trans-chalcone is observed due the existence of the isomerization barrier.

The thermodynamic and kinetics of flavylium based systems as well as their photochromic properties are dramatically dependent on the nature and position of the substituents of the 2-phenylbenzopyrylium core. Anthocyanins are poorly photochromic as the trans-chalcone is a minor species at the equilibrium. In contrast, 3-deoxyanthocyanins such as luteolinidin, exhibit some photochromism since transchalcone (together with quinoidal base) are the main neutral species at moderately acidic medium.[14] The best photochromic systems based on the flavylium multistate of species have been described for simple synthetic flavylium compounds like 4'methoxyflavylium (Fig. 1), 4'-hydroxyflavylium, 7-hydroxyflavylium or 4',7dihydroxyflavylium and similar compounds. ${ }^{[15]}$ However, they are limited in the colour pallet since the trans-chalcones (the ionized trans-chalcones generally lack of photochromic response) are almost colourless and the photoproduct is the yellow flavylium cation. Red shifted absorption bands can be achieved by introducing in the flavylium core amine substituents. This is the case of the compound 4'diethylaminoflavylium previously reported.[16] The photochemical formation of the red flavylium cation was achieved upon irradiation of the metastable $\mathbf{C t}$ and $\mathbf{C t}^{+}$ species.

The flavylium presented in this work (Scheme 1) has one hydroxyl group in carbon 5, allowing the formation of a quinoidal base exhibiting a slight red shifted of its maximum absorption in relation to flavylium cation, extending the colour pallet of the photochemical response. Moreover, micelles are known to modify the $\mathrm{pH}$ distribution of the multistate species and in particular SDS micelles extend the mole fraction 
distribution of the flavylium cation and the quinoidal base to higher $\mathrm{pH}$ values.[17] Introduction of the hydroxyl substituent and the use of SDS micelles, maintains the meta-stability of trans-chalcones, as observed previously for dimethylaminoflavylium, but more significant the one of the quinoidal base, which becomes the photoproduct at more basic $\mathrm{pH}$ values. No negative photochromism is observed in the present system. 


\section{Material and methods}

\section{Reagents}

2,6-dihydroxybenzaldehyde, 98\% was obtained from Ark Pharm (USA), 4'dimethylaminoacetophenone was purchased from Santa Cruz Biochemicals (Netherlands). Sodium dodecyl sulfate (SDS) was purchased Sigma-Aldrich (Madrid, Spain). A universal buffer of Theorell and Stenhagen[18] was prepared dissolving $2.25 \mathrm{~mL}$ of phosphoric acid $85 \%$ (w/w), $7.00 \mathrm{~g}$ of monohydrated citric acid, $3.54 \mathrm{~g}$ of boric acid and $343 \mathrm{~mL}$ of a $1 \mathrm{M} \mathrm{NaOH}$ solution in Millipore water until $1 \mathrm{~L}$.

Synthesis of 5-Hydroxy-4'-Dimethylaminoflavylium

2,6-dihydroxybenzaldehyde $\left(1.4 \times 10^{-3} \quad \mathrm{~mol}, \quad 200 \quad \mathrm{mg}\right)$ and 4'dimethylaminoacetophenone $\left(1.4 \times 10^{-3} \mathrm{~mol}, 235 \mathrm{mg}\right)$ were weighted in a round bottom flask and dissolved in $10 \mathrm{~mL}$ of a mixture of ethyl acetate: methanol (2:1). Then, chlorotrimethylsilane (TMSCl; 5 equiv) was added and the mixture was left to react at room temperature during 5 hours under stirring. To stop the reaction, $30 \mathrm{~mL}$ of water were added and then, the mixture was purified through liquid-liquid extraction using ethyl acetate. The aqueous phase containing mainly the $5 \mathrm{OH} 4$ ' $\mathrm{NMe} 2$ was later purified by column chromatography using LiChroprep ${ }^{\circledR}$ RP-18 (40-63 $\mu$, Merck, Germany). The flavylium compound was recovered with $40 \%$ (v/v) methanol acidified with $2 \% \mathrm{HCl}$, presenting a pink colour. Methanol was evaporated in a rotary evaporator at $38^{\circ} \mathrm{C}$, and the purified $5 \mathrm{OH} 44^{\prime} \mathrm{NMe} 2$ pigment was freeze-dried and stored at $-18^{\circ} \mathrm{C}$ until further analysis.

The formation of the new pigment was followed by HPLC-DAD (Merck), in a reversed-phase $\mathrm{C} 18$ column (Agilent) with $250 \times 4.6 \mathrm{~mm}$ i.d., particle size $2.7 \mu \mathrm{m}$ and at $25^{\circ} \mathrm{C}$. The eluents used were (A) $1 \%(\mathrm{v} / \mathrm{v})$ formic acid in water and (B) $0.5 \%$ 
$(\mathrm{v} / \mathrm{v})$ formic acid in $80 \%(\mathrm{v} / \mathrm{v})$ acetonitrile and the elution gradient was performed from 40 to $85 \% \mathrm{~B}$ during $50 \mathrm{~min}$ at a flow rate of $0.4 \mathrm{~mL} / \mathrm{min}$. After $50 \mathrm{~min}$, the column was washed with $100 \%$ B during 10 min and then it was stabilized with the initial conditions for more $10 \mathrm{~min}$.

The identity and purity of the new flavylium compound was determined by $1 \mathrm{D}$ and 2D NMR experiments $\left({ }^{1} \mathrm{H}, \mathrm{COSY}\right.$, HSQC and $\left.\mathrm{HMBC}\right)$ in $\mathrm{CD}_{3} \mathrm{OD}$ in a Bruker Avance 400 spectrometer.[19]

\section{Determination of the Thermodynamic and Kinetic parameters}

A stock solution $\left(8.0 \times 10^{-4} \mathrm{M}\right)$ of $5 \mathrm{OH} 4$ 'NMe2 in ethanol acidulated with $0.1 \mathrm{M} \mathrm{HCl}$; and a SDS 0.35 M stock solution in Millipore water were prepared. $\mathrm{HCl}, \mathrm{NaOH}$ or Universal buffer were used to adjust the $\mathrm{pH}$ of all the solutions then prepared.

A set of $5 \mathrm{OH} 4$ 'NMe2 solutions in the presence of $0.1 \mathrm{M}$ SDS were prepared in individual $10 \mathrm{~mm}$ pathlength plastic cuvettes for different $\mathrm{pH}$ values along the $\mathrm{pH}$ scale. The pigment was added at the end, and after mixing; the direct $\mathrm{pH}$ jumps kinetics were followed registering the spectral variations in a Varian-Cary 100 Bio and/or 5000 spectrophotometers (Palo Alto, CA, USA) always protecting the solutions from external light sources. This experiment was performed at room temperature and $60^{\circ} \mathrm{C}$. The $\mathrm{pH}$ of the solutions was measured after their preparation in a Radiometer Copenhagen PHM240 pH/77 ion meter.

The determination of the $\mathrm{p} K_{\mathrm{a}}$ was performed from representation of the absorption taken immediately after a direct $\mathrm{pH}$ jump versus $\mathrm{pH}$.

Once the $60^{\circ} \mathrm{C}$ solutions reached the equilibrium (5 days after), a reverse $\mathrm{pH}$ jump was performed adding enough amount of $\mathrm{HCl}$ to reach $\mathrm{pH}=1$. The kinetics were followed in the same way as the direct $\mathrm{pH}$ jumps. 
Titration of trans-chalcones species

A $2.5 \times 10^{-5} \mathrm{M}$ pigment solution in the presence of $0.1 \mathrm{M}$ SDS was prepared in $\mathrm{NaOH}$ $0.1 \mathrm{M}$ (anionic trans-chalcone). Once equilibrated at room temperature (15 minutes after) the solution was titrated with successive additions of small volumes of $\mathrm{HCl}$ and after, the $\mathrm{pH}$ and the spectra of the solution were recorded, always protecting the solutions from external light sources.

\section{Photochemistry assay}

A solution of $5 \mathrm{OH} 4$ ' $\mathrm{NMe} 2\left(2.53 \times 10^{-5} \mathrm{M}\right)$ in the presence of $0.1 \mathrm{M}$ SDS was prepared in $\mathrm{NaOH} 0.1 \mathrm{M}$. Once equilibrated, the solution was placed in a quartz cell $(10 \mathrm{~mm}$ pathlength) and then concentrated $\mathrm{HCl}$, water and universal buffer were added to obtain solutions at $\mathrm{pH} 1,8$ and 10 . The $\mathrm{pH}$ of the final solutions was measured, their initial spectrum was recorded and then each solution was irradiated at $365 \mathrm{~nm}$ under stirring during short periods of time, between each period a spectrum of the solution was recorded, always protecting from external light sources.

\section{Flash Photolysis experiments}

A solution of anionic trans-chalcone of $5 \mathrm{OH} 4$ 'NMe2 $\left(4.5 \times 10^{-5} \mathrm{M}\right)$ in $\mathrm{NaOH} 0.1 \mathrm{M}$ with $0.177 \mathrm{M}$ of SDS was prepared. Once equilibrated, the solution was adjusted to $\mathrm{pH} 1,3,5,7,9$ and 11 using $\mathrm{HCl} 10 \mathrm{M}$ and universal buffer (at desired $\mathrm{pH}$ ). The flash photolysis experiments were performed on a Varian Cary 5000 spectrophotometer equipped with a Harrick fibre-mate (Dunedin, FL, USA) coupled to an Oceanic Optics 4-way cuvette holder (Dunedin, FL, USA). The compartment was isolated from external light sources, the solution placed in a $2 \mathrm{~mm}$ quartz cell and a commercial Achiever 630AF camera flash (Hong Kong, China) was used directly in 
contact with the cell. The kinetics after flash were monitored at the maximum absorption of the flavylium cation or the quinoidal base and their respective chalcones depending on the solution $\mathrm{pH}$.

\section{Results and Discussion}

In spite of the complexity of the system reported in Scheme 1, it can be dramatically simplified considering flavylium cation a di-protic acid defined by eq.(1) and eq.(2), where $\mathrm{CB}, \mathrm{CB}^{-}$are defined as above. ${ }^{\mathrm{d}}$
$\mathbf{A H}^{+} \rightleftharpoons \mathbf{C B}+\mathrm{H}^{+}$
$K^{\prime}{ }_{\mathrm{a}}$
$\mathbf{C B} \rightleftharpoons \mathbf{C B}^{-}+\mathrm{H}^{+}$
$K^{\prime}{ }_{\text {a }}$

Where:

$$
\begin{aligned}
& {[\mathbf{C B}]=[\mathbf{A}]+[\mathbf{B}]+[\mathbf{C c}]+[\mathbf{C t}] ;} \\
& {\left[\mathbf{C B}^{-}\right]=\left[\mathbf{B}^{-}\right]+\left[\mathbf{C c}^{-}\right]+\left[\mathbf{C t}^{-}\right]} \\
& K_{a}^{\prime}=K_{a}+K_{h}\left(1+K_{t}\right)+K_{h} K_{t} K_{i} \\
& K^{\prime \prime}{ }_{a}=\frac{K_{A / A} K_{a}+K_{B / B} K_{h}+K_{C c / C c} K_{h} K_{t}+K_{C t / C t} K_{h} K_{t} K_{i}}{K_{a}^{\prime}}
\end{aligned}
$$

\section{$5 O H 4$ 'NMe2 in the presence of SDS micelles}

In spite of the existence of a significant aggregation of this compound in pure water, even at low concentrations, see supplementary material, this phenomenon was not observed in the presence of SDS micelles.

The absorption spectra of $5 \mathrm{OH} 4{ }^{\prime} \mathrm{NMe} 2\left(2.56 \times 10^{-5} \mathrm{M}\right)$ in the presence of SDS micelles (0.1 M) taken immediately after direct $\mathrm{pH}$ jumps are shown in Fig. 2. There is an equilibrium between $\mathbf{A H}^{+}$and $\mathbf{A}$ with $\mathrm{p} K_{\mathrm{a}}=7.6$, Fig. 2a. These two species give rise to

\footnotetext{
${ }^{\mathrm{d}}$ In the present work the species $\mathrm{Cc}$ and $\mathrm{B}$ as well as the respective anionic forms are not observed at the equilibrium or in the metastable states. They are transient species.
} 
a metastable state whose $\mathrm{pH}$ limits will be defined bellow. For very basic solutions it is necessary to heat the system at $60{ }^{\circ} \mathrm{C}$ in order to observe the kinetic processes towards the equilibrium, Fig. $\mathbf{2 b}$ and Fig. 2c. Only at very basic pH values, for example $\mathrm{pH}=13$, the anionic form of the trans-chalcone is observed in a few minutes at room temperature, similarly to the behaviour of other flavylium systems bearing amine substituents. ${ }^{\text {Error! Bookmark not defined. }}$

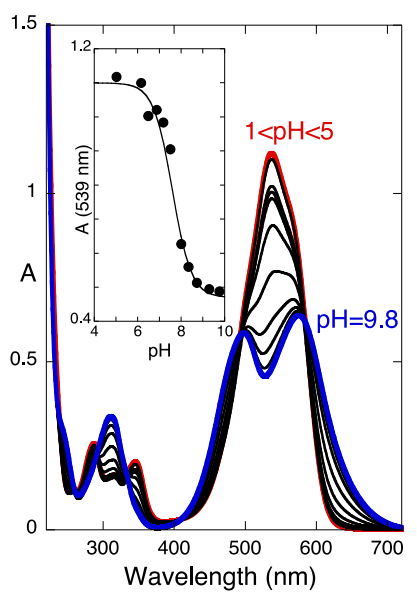

(a)

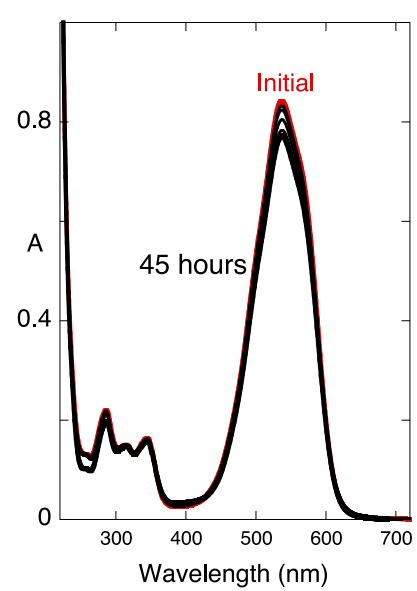

(b)

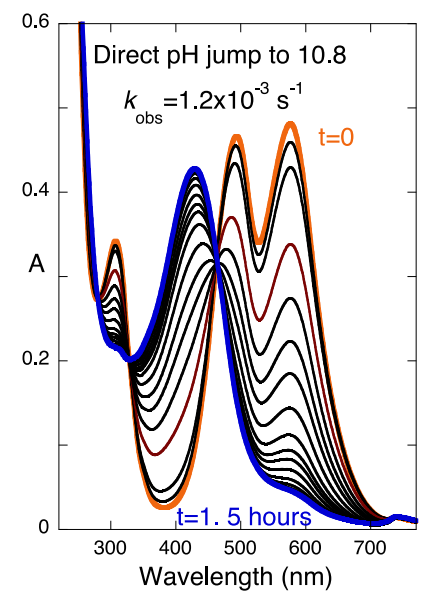

(c)

Figure 2. (a) Spectral variations of $5 \mathrm{OH} 4$ ' $\mathrm{NMe} 2\left(2.56 \times 10^{-5} \mathrm{M}\right)$ in the presence of SDS micelles $(0.1$ M) taken immediately after a direct $\mathrm{pH}$ jump. The results indicate that the flavylium cation is in equilibrium with the quinoidal base with $\mathrm{p} K_{\mathrm{a}}=7.6$; (b) Spectral variations at $\mathrm{pH}=6.8$ at $60{ }^{\circ} \mathrm{C}$ after 45 hours; (c) Spectral variations upon a direct $\mathrm{pH}$ jump to $\mathrm{pH}=10.8$ at $60{ }^{\circ} \mathrm{C}$ after $90 \mathrm{~min}$.

More information regarding the meta-stability of the flavylium cation-quinoidal base system was achieved by carrying out a series of direct $\mathrm{pH}$ jumps at $60{ }^{\circ} \mathrm{C}$, represented in Fig. 3. The absorbance of the flavylium cation after 5 days at $60{ }^{\circ} \mathrm{C}$ shows an apparent $\mathrm{p} K_{\mathrm{a}}=7.5$. The quinoidal base completely disappears and at higher $\mathrm{pH}$ values the absorption spectra identified as trans-chalcones are dominant (see below). After 5 days at $60{ }^{\circ} \mathrm{C}$ some decomposition occurs. The $\mathrm{pH}$ dependent rate of the quinoidal base disappearance, after the direct $\mathrm{pH}$ jumps to basic medium at $60{ }^{\circ} \mathrm{C}$, is shown in Fig. 3c. Similarly, to what was observed for other flavylium systems the quinoidal 
base is attacked by $\mathrm{OH}^{-}$and the respective hydration rate is directly proportional the hydroxyl concentration, $2.5\left[\mathrm{OH}^{-}\right] \mathrm{s}^{-1}$.

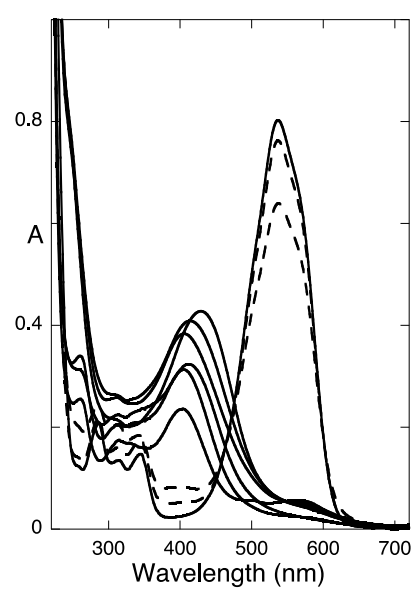

(a)

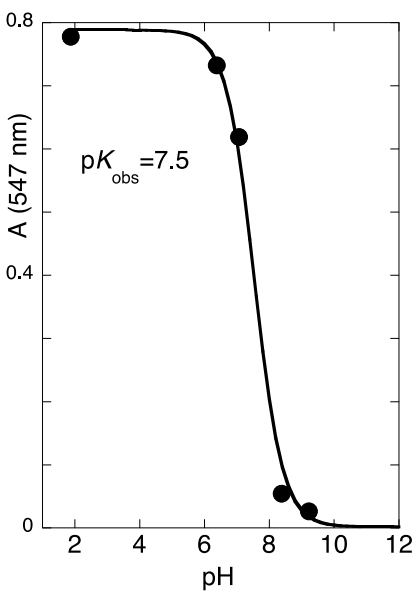

(b)

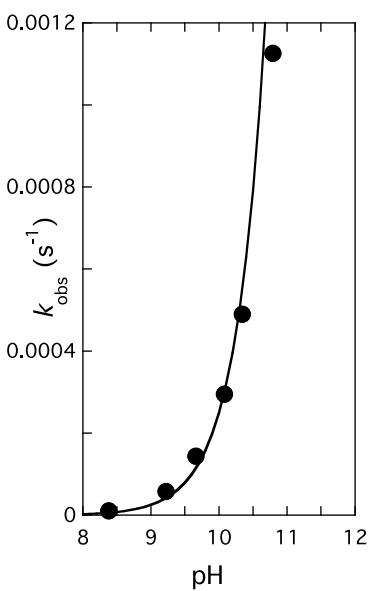

(c)

Figure 3. (a) Spectral variations of the compound $5 \mathrm{OH} 44^{\mathrm{NMe}} 2\left(2.56 \times 10^{-5} \mathrm{M}\right)$ after a direct $\mathrm{pH}$ jump $\left(60^{\circ} \mathrm{C}\right.$ after 5 days). The traced lines correspond to $\mathrm{pH}$ around 7 where stability of the solution was not yet achieved (traced lines); (b) Representation of the flavylium cation absorption in (a) at $547 \mathrm{~nm}$ as a function of $\mathrm{pH}$; (c) Rate constants of the quinoidal base disappearance in basic medium at $60^{\circ} \mathrm{C}$. Fitting was achieved for $2.5\left[\mathrm{OH}^{-}\right] \mathrm{s}^{-1}$.

The anionic trans-chalcone was prepared at very basic pHs and from this species a series of reverse $\mathrm{pH}$ jumps were carried out, Fig. $4 .{ }^{\mathrm{e}}$ Considering the behaviour of other flavylium compounds possessing amine substituents, the first protonation constant, $\mathrm{p} K_{\mathrm{Ct}+/ \mathrm{Ct}}=2.6 \pm 0.1$, can be attributed to the acid-base equilibrium between the protonated trans-chalcone and the neutral one. The protonated species is stabilized by the negatively charged surface of the SDS micelles. The second equilibrium, $\mathrm{p} K_{\mathrm{Ct} / \mathrm{Ct}-}$ $=10.7 \pm 0.1$, is attributed to the deprotonation of the trans-chalcone to give its anionic form. The formation of the negatively charged trans-chalcone, is not favoured by the negatively charge of the SDS micelles surface and thus takes place only at very basic

\footnotetext{
e Special care should be taken to avoid interference of the photochemical reactions.
} 
$\mathrm{pH}$ values. The trans-chalcones are meta-stable at room temperature unless for very basic solutions.

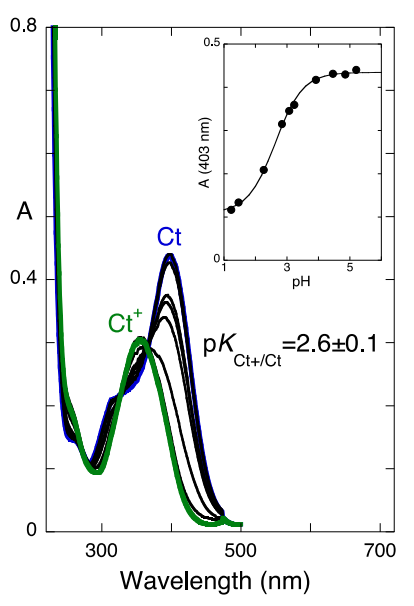

(a)

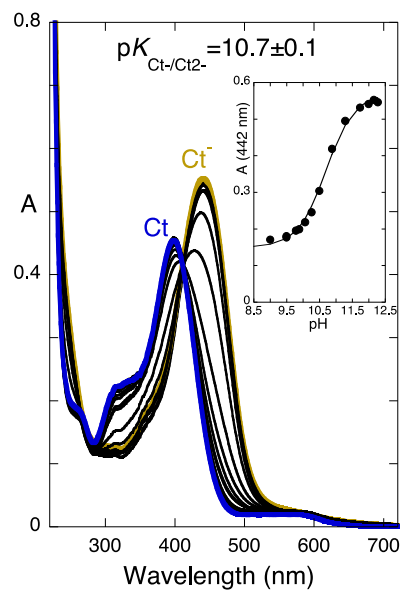

(b)

Figure 4. Titration of the trans-chalcones of the compound 5OH4'NMe2 from $\mathrm{pH}=13$ : (a) $1<\mathrm{pH}<5$; (b) $8.5<\mathrm{pH}<12.5$. The experiments were performed in the dark to prevent the photochromic response of the trans-chalcones. The solutions are meta-stable at room temperature unless for very basic $\mathrm{pH}$ values.

The limits of the meta-stability of the trans-chalcones was also studied, Fig. 5, by following the spectral variations of the reverse $\mathrm{pH}$ jumps shown in Fig. 4 at $60{ }^{\circ} \mathrm{C}$. The flavylium cation is formed and equilibrates with trans-chalcones (neutral and protonated), with $\mathrm{p} K_{\mathrm{obs}}=4.1$.

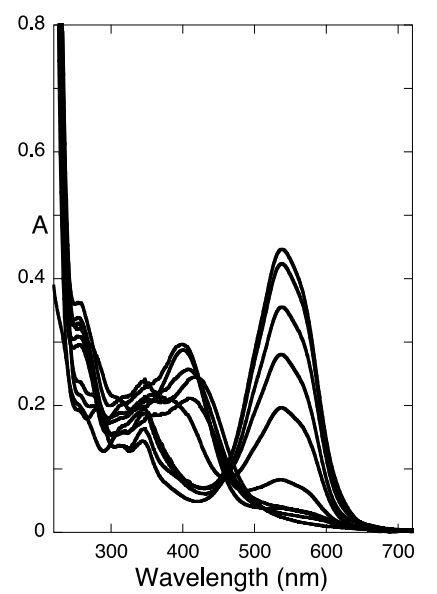

(a)

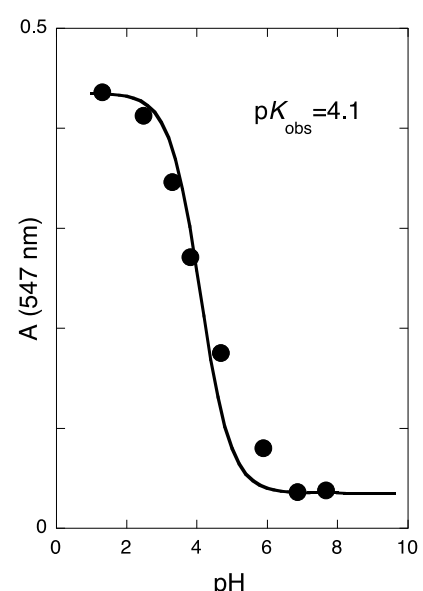

(b) 
Figure 5. (a) Spectral variations of the compound 5OH4'NMe2 after a series of reverse $\mathrm{pH}$ jumps from the anionic trans-chalcone equilibrated at $\mathrm{pH}=13$ after 5 days at $60{ }^{\circ} \mathrm{C}$; (b) Absorbance of the flavylium compound at $547 \mathrm{~nm}$ versus $\mathrm{pH}$. The inflection point occurs for $\mathrm{p} K_{\mathrm{obs}}=4.1$

The absorption spectra after 5 days at $60{ }^{\circ} \mathrm{C}$ obtained from direct $\mathrm{pH}$ jumps of equilibrated solutions at $\mathrm{pH}=1$, Fig. 3a, and reverse $\mathrm{pH}$ jumps from anionic transchalcone, Fig. 5a, are similar but not coincident. The most interesting feature is however the observed $\mathrm{p} K_{\mathrm{a}}$ of the flavylium cation: $\mathrm{p} K_{\mathrm{obs}}=4.1$ (Fig. 5b) that compares with $\mathrm{p} K_{\mathrm{obs}}=7.5$ (Fig. 3b). The question is which of the two values is closer to the putative equilibrium that even at $60{ }^{\circ} \mathrm{C}$ we are not able to define with precision. In both cases, the quinoidal base disappears completely, confirming its metastable state. Considering that the equilibrium between flavylium cation and the neutral species is given by eq.(3), the value $\mathrm{p} K_{\mathrm{obs}}=7.5$ would imply the flavylium cation and the quinoidal base as the major neutral species, circa 95\% and lack of the others in particular the trans-chalcone, which is not the observed behaviour in Fig. 3a, see in supplementary material the respective mathematical demonstration. On the other hand, the value $\mathrm{p} K_{\mathrm{obs}}=4.1(0.2 \%$ of flavylium cation and quinoidal base $)$ is compatible with a system where trans-chalcone is dominant and quinoidal base is negligible. For practical purposes we assign the value $\mathrm{p} K_{\mathrm{obs}}=4.1$ as representing the equilibrium, but it cannot be excluded that this mole fraction distribution is another meta-stable state reached from the trans-chalcones. 


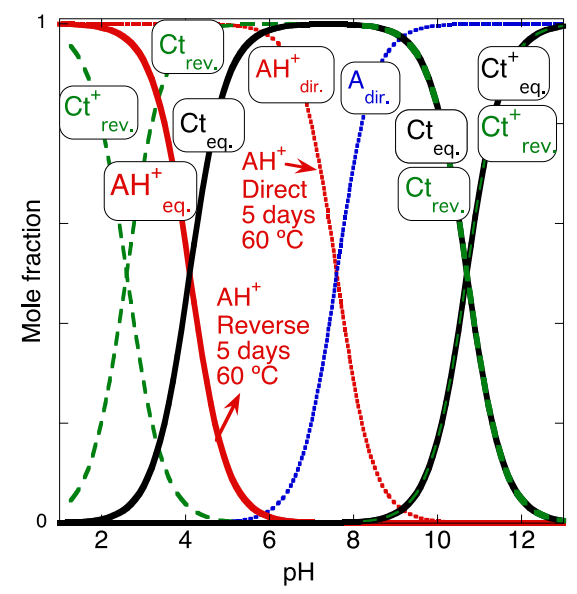

Scheme 2. Mole fraction distribution of the flavylium cation (red-pointed line) and quinoidal base (blue-pointed line) after a direct $\mathrm{pH}$ jump from equilibrated solutions at $\mathrm{pH}=1$; mole fraction distribution after a reverse $\mathrm{pH}$ jump from the stable anionic trans-chalcone at $\mathrm{pH}=13$ (green traced lines); flavylium cation (red-full line), trans-chalcone (black full line) and anionic trans-chalcone (black full line). For $\mathrm{pH}>9$ the equilibrium distribution is coincident with the one of the reverse $\mathrm{pH}$ jumps.

Scheme 2 summarizes the behaviour of this compound. It can be observed that a meta-stable state is achieved after a direct $\mathrm{pH}$ jump from equilibrated solutions of the flavylium cation. Only two species are observed, the flavylium cation (red-pointed line) and the quinoidal base (blue-pointed line) with a $\mathrm{p} K_{\mathrm{a}}=7.5$. The extended $\mathrm{pH}$ range of the flavylium cation is attributed to the electrostatic effect that stabilizes the flavylium cation.(meter J. C. Lima, C. Vautier-Giongo, A.Lopes, E. Melo,

F. H. Quina, A. L. Maçanita, Color Stabilization of Anthocyanins: Effect of SDS Micelles on the Acid-Base and Hydration Kinetics of Malvidin 3-Glucoside (Oenin), J. Phys. Chem. A 2002, 106, 5851-5859).

Similarly to anthocyanins and other related compounds, the quinoidal base does not hydrate but reacts with $\mathrm{OH}^{-}$at higher $\mathrm{pH}$ values. ${ }^{\text {Error! Bookmark not defined. However, in }}$ the present case this reaction is extremely slow unless for very high $\mathrm{pH}$ values. The 
titration of the anionic trans-chalcone (reverse $\mathrm{pH}$ jumps) in dark conditions leads to the other metastable system, represented in Scheme 2 by the green traced lines.

The evolution of the absorption spectra of the metastable species resulting from direct $\mathrm{pH}$ jumps $\left(\mathbf{A H}^{+}\right.$and $\left.\mathbf{A}\right)$ as well as those from the reverse $\mathrm{pH}$ jumps $\left(\mathbf{C t}^{+}, \mathbf{C t}\right.$ and $\left.\mathbf{C t}^{-}\right)$ after 5 days at $60{ }^{\circ} \mathrm{C}$ indicate that the quinoidal base disappears in the former to give the trans-chalcone or its anionic form (depending on $\mathrm{pH}$ ) and the protonated transchalcone disappears to give the flavylium cation. The neutral trans-chalcone equilibrates with flavylium, $\mathrm{p} K_{\mathrm{obs}}=4.1$, and it is the dominant equilibrium species in the $\mathrm{pH}$ interval $5<\mathrm{pH}<9$, when starts to equilibrate with the anionic trans-chalcone at higher $\mathrm{pH}$ values. The $\mathrm{pH}$ domain of the flavylium cation at the equilibrium, Scheme 2 , is reduced because trans-chalcone that is slowly formed is solubilized in the more hydrophobic region of the SDS micelles.

A final remark to mentioned that the double-charged flavylium cation was observed at 6M (HCL) (Paulinha confirmar) but not the dianionic trans-chalcone, probably due to the electrostatic repulsion of the negatively charged species.

\section{The photochromic system}

The spectral variations upon irradiation of the metastable trans-chalcones of the compound 5OH4'NMe2 (obtained from protonation of the stable anionic transchalcone at $\mathrm{pH}=13$ ) for three representative $\mathrm{pH}$ values are shown in Fig. 6.

At $\mathrm{pH}=1.5$ it is the metastable protonated trans-chalcone that absorbs light and the photoproduct is the flavylium cation. At $\mathrm{pH}=8.4$ and 10.1 it is essentially the neutral trans-chalcone, that absorbs light and give a mixture of flavylium cation and quinoidal base (at $\mathrm{pH}=8.4$ ) and quinoidal base (at $\mathrm{pH}=10.1$ ). The photoproduct quinoidal base is a metastable photoproduct. 


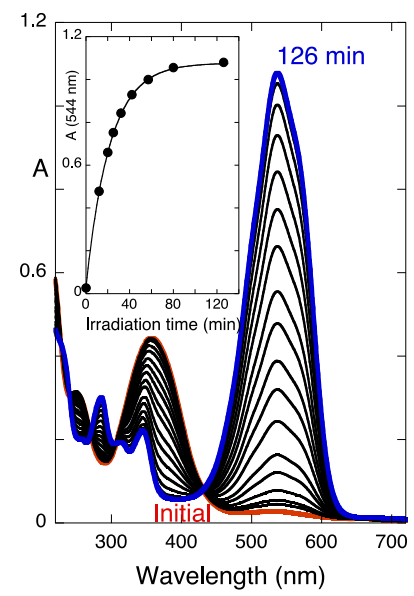

(a)

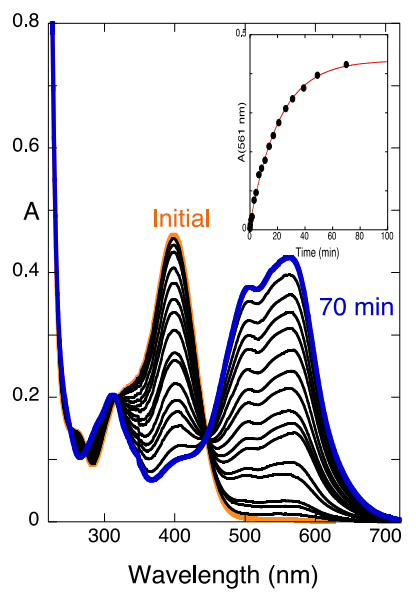

(b)

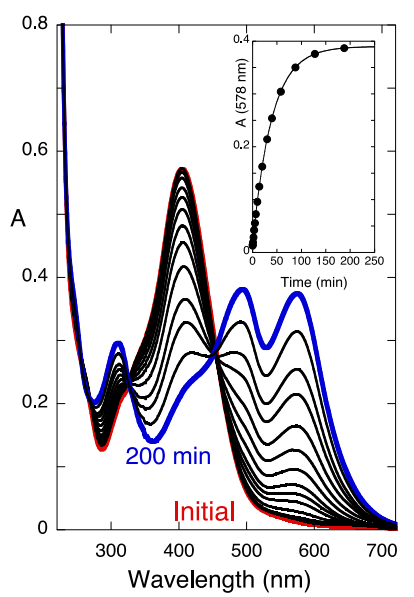

(c)

Figure 6. (a) Irradiation at $365 \mathrm{~nm}\left(\mathrm{I}_{0}=2.8 \times 10^{-7}\right.$ Einstein $\left.\mathrm{min}^{-1}\right)$ of the metastable trans chalcone at $\mathrm{pH}=1.5,2.6 \times 10^{-5} \mathrm{M} ;(\mathbf{b})$ the same at $\mathrm{pH}=8.4,2.5 \times 10^{-5} \mathrm{M} ;(\mathbf{c})$ the same at $\mathrm{pH}=10.1,2.8 \times 10^{-5} \mathrm{M}$.

Flash photolysis in the domain of seconds[20] was performed for representative $\mathrm{pH}$ values. The results at $\mathrm{pH}=3$ and $\mathrm{pH}=10$ are shown in Fig.7a and Fig. $\mathbf{7 b}$ respectively. In the $\mathrm{pH}$ interval $1<\mathrm{pH}<9$, the behaviour is the same of the one at $\mathrm{pH}=3$. In Fig. 7a two successive flashes were applied to the solution. At $540 \mathrm{~nm}$ (flavylium cation absorption) there is a sudden increasing of the absorption while at $390 \mathrm{~nm}$ (transchalcone absorption) a concomitant bleaching. This means that during the resolution time of this flash photolysis $(0.1 \mathrm{~s})$ the flavylium cation is already formed at the expenses of trans-chalcone. The light absorption promotes the photo-induced isomerization and the primary photoproduct (cis-chalcone) should give the flavylium cation via tautomerization reaction followed by the de-hydration, Scheme 1. These processes are concluded before $0.1 \mathrm{~s}$. At $\mathrm{pH}=10$ formation of the quinoidal base is relatively slow and the system reverts back thermally to the equilibrium. It is similar to the previous photochromic systems described in the introduction, Fig. 1b, but takes place at much higher pHs. 

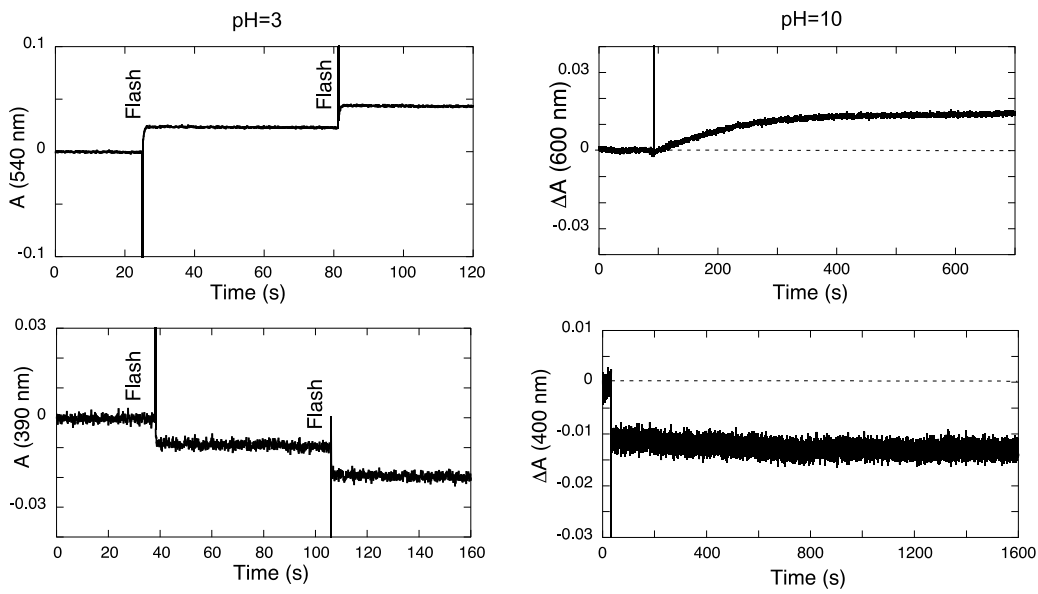

Figure 7. Flash photolysis in the domain of seconds of the compound 5OH4'NMe2: (a) at $\mathrm{pH}=3$; (b) at $\mathrm{pH}=10$.

In Scheme 3 the photochromic system is summarized. At lower $\mathrm{pH}$ values it is the metastable protonated trans-chalcone that yields the flavylium cation as described in Fig. 1a for 4'-methoxyflavylium. At $\mathrm{pH}=10.1$ trans-chalcones are still the dominant species and the photoproduct is the metastable quinoidal base.

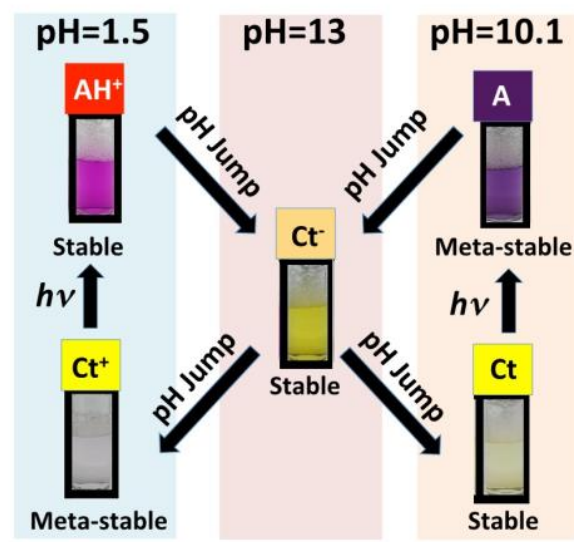

(a)

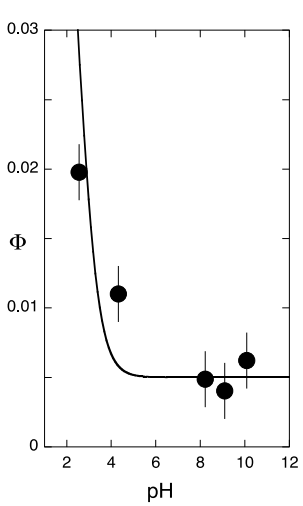

(b) 
Scheme 3. (a) A photochromic system from a metastable state to give a stable photoproduct can be prepared at $\mathrm{pH}=1.5$. The contrary is obtained at $\mathrm{pH}=10.1 . \mathrm{Ct}^{-}$is thermal and photochemical stable; (b) Quantum yields as a function of $\mathrm{pH}$. The quantum yields are roughly fitted for $\mathrm{p} K=2.6\left(\mathrm{Ct}^{+} / \mathrm{Ct}\right)$ and $\Phi=0.05$ and $\Phi=0.005$ respectively for $\mathrm{Ct}^{+}$and $\mathrm{Ct}$.

Several years ago, we introduced the energy level diagram to account for the details of the flavylium based multistates.[5, 21] In Scheme 4, a semi-quantitative proposal is shown for $5 \mathrm{OH} 4 \mathrm{NMe} 2$, because it was not possible to clearly define the equilibrium (in grey colour the species not defined with precision). The quantitative energy level of 4'-hydroxyflavylium is shown for comparison purposes.[22]

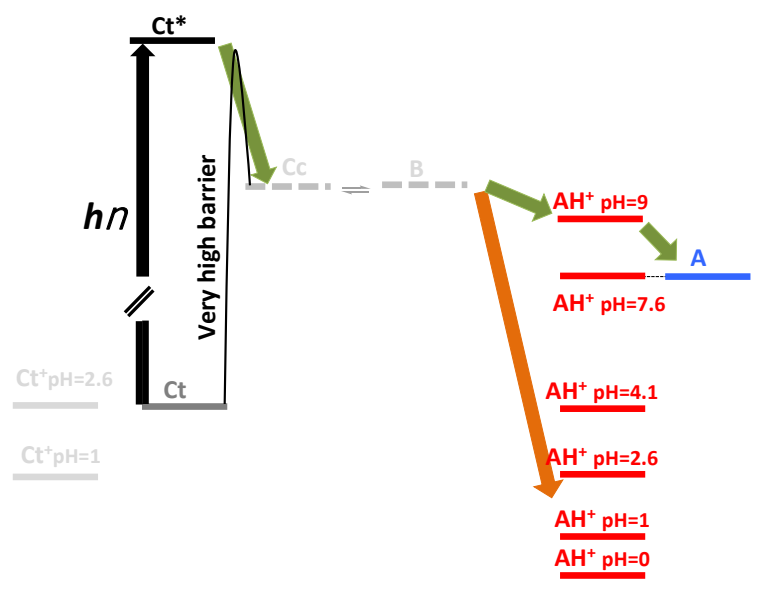

(a)

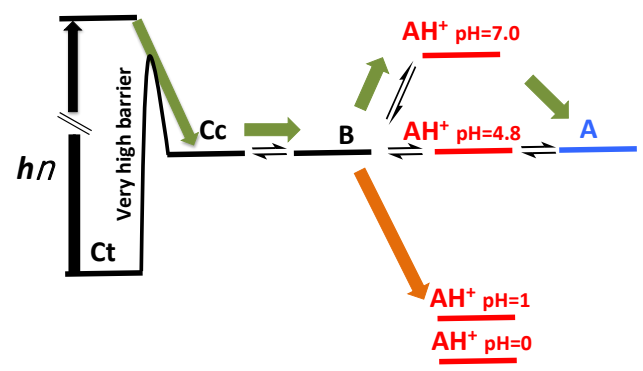

(b)

Scheme 4. (a) Qualitative energy level diagram of 5OH4'NMe2 in the presence of SDS micelles; (b) Quantitative energy level diagram of 4'-hyydroxyflavylium for comparison purposes.[22] 
The interpretation for the unusual photochromic signal of the quinoidal base at basic $\mathrm{pH}$ values seems to be linked to the high energy level of $\mathbf{C c}$ and $\mathbf{B}$. As shown in Scheme 4a, after a direct $\mathrm{pH}$ jump to $\mathrm{pH}=9$, the primary photoproduct gives spontaneously the quinoidal base, while in 4'-hydroxyflabylium, the primary photoproduct $\mathbf{C c}$ equilibrates fast with $\mathbf{B}$ and more slowly with $\mathbf{A}$. The diagram in Scheme 4a explains also the meta-stability of $\mathbf{A}$ upon a direct $\mathrm{pH}$ jump. A once formed to reach the more stable $\mathbf{C t}$, needs to overcome a high energy barrier, since the system should pass through $\mathbf{A} \mathbf{H}^{+}, \mathbf{B}$ and $\mathbf{C c}$.

\section{Conclusions}

The photochromic systems based on the flavylium multistate of species are extremely versatile. The nature and position of the substituents can modify not only the kinetics and thermodynamic of the system but also the photochromic response. In particular the colour pallet of the photochromic products could be extended by the amine substituents. Moreover, the use of micelles and other hosts can dramatically modify the mole fraction distribution of the flavylium multistate species, in particular extending the $\mathrm{pH}$ domain of the photoactive trans-chalcone. In the present work the conjugation of the selected substituents and their position together with the use of SDS micelles allowed to define a new flavylium based photochromic system exhibiting positive photochromism in an extended $\mathrm{pH}$ region, $1<\mathrm{pH}<10$. In spite of the low quantum yields of reaction, the performance of the system relatively to the appearance of the colour is good due to the very high molar absorption coefficients of the final photoproducts.

\section{Acknowledgements}


This work was supported by the Associated Laboratory for Sustainable

Chemistry, Clean Processes and Technologies LAQV through the national funds from UIDB/50006/2020. It was also supported by the project PTDC/QUI-OUT/29013/2017 funded by FCT and FEDER. P.A. and A.R.P. gratefully acknowledge their doctoral grants from FCT (SFRH/BD/143309/2019 and SFRH/BD/146549/2019, respectively). J.M. is grateful for a doctoral grant from CONACYT (MEX/Ref. 288188). J.O. would like to thank the FCT for her IF contract (IF/00225/2015) and I.F for her research contract (SFRH/BPD/86173/2012).

\section{References}

[1] F. Pina, M.J. Melo, C.A.T. Laia, A.J. Parola, J.C. Lima, Chemistry and Applications of Flavylium Compounds: a Handful of Colours, Chem. Soc. Rev. 41 (2012) 869-908.

[2] R. Brouillard, J.E. Dubois, Mechanism of Structural Transformations of Anthocyanins in acidic media, J. Am. Chem. Soc. 99 (1977) 1359-1364.

[3] R. Brouillard, J. Lang, The Hemiketal-cis-Chalcone Equilibrium of Malvin, a Natual Anthocyanin, Can. J. Chem.-Rev. Can. Chim. 68 (1990) 755-761.

[4] R.A. McClelland, S. Gedge, Hydration of the Flavylium Ion J. Am. Chem. Soc. 102 (1980) 58385848 .

[5] F. Pina, Chemical Applications of Anthocyanins and Related Compounds. A Source of Bioinspiration, J. Agric. Food Chem. 62 (2014) 6885-6897.

[6] R. Brouillard, B. Delaporte, J.E. Dubois, Chemistry of Anthocyanins Pigments.3. Relaxation Amplitudes in pH-Jump Experiments, J. Am. Chem. Soc. 100 (1978) 6202-6205.

[7] A.L. Macanita, P.F. Moreira, J.C. Lima, F.H. Quina, C. Yihwa, C. Vautier-Giongo, Proton Transfer in Anthocyanins and Related Flavylium Salts. Determination of Ground-State Rate Constants with Nanosecond Laser Flash Photolysis, J. Phys. Chem. A 106 (2002) 1248-1255.

[8] L. Jurd, T.A. Geissman, Anthocyanins and Related Compounds.2. Structural Transformations of Some Anhydro Bases Journal of Organic Chemistry 28 (1963) 2394-\&.

[9] R. Matsushima, K. Miyakawa, M. Nishihata, Photochromic Properties of 2-hydroxychalcones Chem. Lett. (1988) 1915-1916.

[10] R. Matsushima, H. Mizuno, A. Kajiura, Convenient Chemical Actimometer with 2-hydroxy-4'Methoxychalcone B Chem Soc Jpn 67 (1994) 1762-1764.

[11] P. Figueiredo, J.C. Lima, H. Santos, M.C. Wigand, R. Brouillard, A.L. Macanita, F. Pina, Photochromism of the Synthetic 4',7-Dihydroxyflavylium Chloride J. Am. Chem. Soc. 116 (1994) 1249-1254.

[12] F. Pina, M. Maestri, V. Balzani, Photochromic flavylium compounds as multistate/multifunction molecular-level systems, Chem. Commun. (1999) 107-114.

[13] F. Pina, M.J. Melo, M. Maestri, P. Passaniti, V. Balzani, Artificial chemical systems capable of mimicking some elementary properties of neurons, J. Am. Chem. Soc. 122 (2000) 4496-4498.

[14] M.J. Melo, S. Moura, A. Roque, M. Maestri, F. Pina, Photochemistry of luteolinidin - "Writelock-read-unlock-erase" with a natural compound, J. Photochem. Photobiol. 135 (2000) 33-39.

[15] F. Pina, M.J. Melo, A.J. Parola, M. Maestri, V. Balzani, pH-controlled photochromism of hydroxyflavylium ions, Chem. Eur. J. 4 (1998) 2001-2007. 
[16] A. Roque, C. Lodeiro, F. Pina, M. Maestri, S. Dumas, P. Passaniti, V. Balzani, Multistate/multifunctional systems. A thermodynamic, kinetic, and photochemical investigation of the 4 '-dimethylaminoflavylium compound, J. Am. Chem. Soc. 125 (2003) 987-994.

[17] A. Roque, F. Pina, S. Alves, R. Ballardini, M. Maestri, V. Balzani, Micelle effect on the 'writelock-read-unlock-erase' cycle of 4 '-hydroxyflavylium ion, J. Mater. Chem. 9 (1999) 2265-2269.

[18] Küster FW, Thiel A. Tabelle per le Analisi Chimiche e Chimico- Fisiche. 12 th ed Milano, Italy: Hoepli; 1982. p. 157-60.

[19] d.H.H.-p. . 1H - H-3: 8.08 ppm, d (9.2 Hz); H-6: 7.03 ppm, d (8.1 Hz); H-7: 7.79 ppm, t (8.1 Hz); H-8: 7.36 ppm, d (8.1 Hz); H-2',6': 8.30 ppm, d (8.8 Hz); H-3',5': 7.02 ppm, d (8.8 Hz); H-4'-N(CH3)2: 3.32 ppm, s., H.-. 13C - C-2: 171.0 ppm (HMBC, H-4 and H-2',6'); C-3:113.7 ppm (HSQC, H-3); C-4: 142.8 ppm (HSQC, H-4); C-4a: 154.7 ppm (HMBC, H-4, H-6 and H-8); C-5: 156.3 ppm (HMBC, H-4, H-6 and H-7); C-6: 111.7 ppm (HSQC, H-6); C-7: 136.9 ppm (HSQC, H-7); C-8: 107.6 ppm (HSQC, H-8); C-8a: 154.6 ppm (HMBC, H-8); C-1': 157.4 ppm (HMBC, H-2',6'); C-2',6': 133.5 ppm (HSQC, H-2',6'); C-3',5': 113.6 ppm (HMBC, H-3',5'); C-4':157.6 ppm (HMBC, H-N-CH3); C-4'$\mathrm{N}-(\mathrm{CH} 3) 2: 39.9$ ppm (HSQC, H-4'-N-(CH3)2).

[20] M. Maestri, R. Ballardini, F. Pina, M.J. Melo, An easy and inexpensive flash spectroscopy experiment, J. Chem. Educ. 74 (1997) 1314-1316.

[21] F. Pina, M.J. Melo, M. Maestri, R. Ballardini, V. Balzani, Photochromism of 4'-methoxyflavylium perchlorate. A "write-lock-read-unlock-erase" molecular switching system, J. Am. Chem. Soc. 119 (1997) 5556-5561.

[22] J. Mendoza, N. Basilio, V. de Freitas, F. Pina, New Procedure To Calculate All Equilibrium Constants in Flavylium Compounds: Application to the Copigmentation of Anthocyanins, ACS Omega 4 (2019) 12058-12070. 